\title{
An interactive video database for food engineering education
}

\author{
Yuanyuan $\mathrm{Jia}^{1,}{ }^{1,}$, Wei Fu ${ }^{1}$, Zheng Zhao ${ }^{2}$ \\ ${ }^{1}$ College of Material Science \& Chemical Engineering, Tianjin University of Science and \\ Technology, Tianjin, 300457, China \\ ${ }^{2}$ College of Food Engineering \& Biological Technology, Tianjin University of Science and \\ Technology, Tianjin, 300457, China
}

aemail: jiayuanyuan55@hotmail.com

\begin{abstract}
Keywords: Interactive Video System; E-learning; Bilingual Video Resources; Automatic Learning; Inquiry-based Learning
\end{abstract}

\begin{abstract}
The advantage of videos in food engineering education has been well-recognized. An interactive video system was developed to guide students' automatic learning, covering the subjects of food engineering, chemical engineering, mechanical engineering, biological engineering, automation engineering, and basic experiments. It takes the mode of 'inquiry-based learning'. Quizzes were given to each video aiming at the key points of the contents. Besides, students may raise questions via a mail box. Students respond that the system benefits their E-learning, which affords learners more flexibility.
\end{abstract}

\section{Introduction}

Video has the advantage of strong performance. American biologist Medina, focusing on gene research of human brain, pointed out that sense organs always work together. A beneficial effect can be obtained providing that several organs can active up during the learning process; among them, vision is the most active sense organ. The more input information in forms of picture, the easier it will be to be identified and recalled.

In recent years, people put more and more attention on taking advantage of video to promote education and teaching. For example, there is a program of 'Teaching Videos from Famous Universities' on the Chinese mainstream video website 'Youku (http://www.youku.com/), which displays many teaching videos and lectures from Chinese first-class universities. The 'video open class' website (http://www.icourses.edu.cn/) organized by Chinese Ministry of Education, already on line for one year, has launched more than one hundred courses.

We have built up a video database of food engineering education in the practice of developing the National Excellence Course 'Principles of Food technology' [1] and the National Bilingual Demonstration Course 'Introduction to Food Science and Technology' [2, 3]. On the two course websites, we supply 541 on-line videos, spreading over 19 files, such as 'Food Overview', 'Dairy Processing', 'Meat and Poultry Processing', 'Baking and Pasta', 'Food and Culture' and so on. We also set up a video database [4] on the public educational service platform of Tianjin University of Science and Technology, as well as on 'Youku' website. Food videos have been utilized in class instruction, experiment instruction, internship, and extracurricular practice. It can fill the gap of teaching hours, laboratory facilities and practice sites to some extent. Food videos hold the promise of establishing imaginary thinking and widening student's sight.

As constructivism learning theory saying, abundant resources should be provided for the students' individualized learning, independent learning and cooperative learning. Course materials should meet the students' requirement and learning foundation, so as to facilitate automatic learning. That is to say, students absorb and digest automatically, so that they can develop their own cognitive structure.

Education Department of the Ministry of Education issued the 'Teaching Requirements for College English (for trial implementation)' on Feb 2004, which raised a new trend of college English teaching reforms. Emphasizing on students' learning autonomy is one of the important aspects of the activities. After years of efforts, the autonomic learning in foreign language teaching has received a significant effect. Foreign language teaching gained great progress in the construction and utilization [5, 6, and 7] of teaching videos, supplying a lot of experience for the 
establishment of scientific video resources. In our bilingual class of professional courses, the videos can also play double roles in assisting teaching and language learning. Enriching learning resources including scientific videos and leading students to apply learning resources are the important facets of automatic learning.

\section{Purpose for the System}

We have done a lot on the construction of video resources and the accumulation of a wealth of video materials, which is a good promotion to expanding students' vision and learning space as well as supplementing practice resources. However, when students study with the video database by themselves, they can't reach the expected effect, for lack of guidance from teachers. In particular, most of the videos we provide are interpreted in English, such as the series of videos 'How it's made' from the United States Discovery Communication Corporation etc., and most of the students say that they have certain difficulties for their self-study. One study shows that $88 \%$ of the normal university students hope to get teacher's guidance when they are learning the case videos. Consequently, we established an interactive video database, selecting a number of well-made, typical videos illustrating advanced technologies. Teachers put forward several questions and give out answers aiming at the key points for each video. At the same time, we expand this interactive video system from food engineering to chemical engineering, biological engineering, mechanical engineering and automation engineering.

\section{Layout and Design for the System}

The principle of a website's layout design lies in its practicability and high efficiency. We try to make the pages elegant and distinct, with fresh hue and simple structure. The navigation bar is located on the top of the page and the professional fields concerned are located at the bottom. The colors of the navigation bar, texts and pictures, etc. are determined for the sake of harmony. The web page takes blue-white tone, and has both Chinese and English version, see Figure 1.

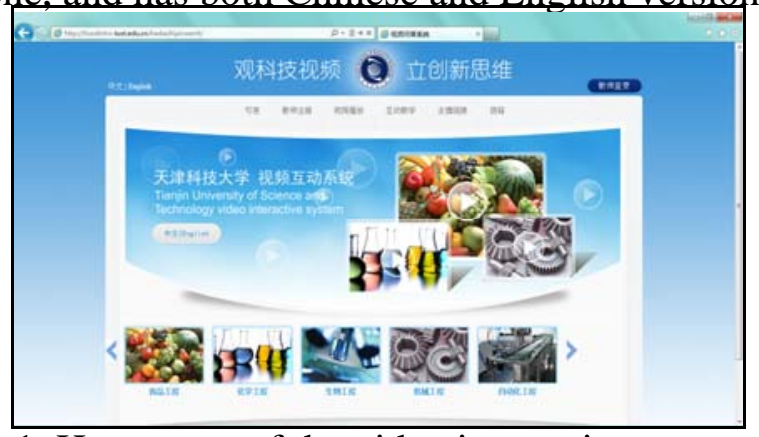

Fig.1. Homepage of the video interactive system

\section{Content of the Video Interactive System}

The navigation bar has 6 menus, which are Introduction, Teacher registration, Video playing, Interactive teaching, Links, and message board.

The video resources of 'video playing' are mainly collected from the website 'Food science and technology introduction', which covering Food engineering, Chemical engineering, Biological engineering, Mechanical engineering, Automation engineering and Basic experiments. Each subject has several subtitles, for example, 'Food engineering' has three subtitles, Food security, Food processing and Food science. These videos are primarily collected from the following websites: (1) 'How it's made' program of the USA Discover Channel; (2)The agriculture program of CCTV-7; (3) the column of 'Development of food products' (From concept to consumer: Food product development) and 'Films of food preservation' (The great fight for food preservation) from the official website of Institute of Food Technology (IFT); (4)'Candy and food packing' videos played on the 'Manufacture Festival' of Stanford University; (5) The serial videos 'Food Security' played on the website of USA Food and Drug Administration; (6) 'Space Food' videos launched by Food technology and commercial center of National Aeronautics and Space Administration of USA. What's more, the propaganda videos and animations made by enterprises' websites are also inclusive. For example, there are 15 videos introducing the separation equipment and their 
application published by Westfalia, the multinational corporation. Besides, Koch, the world famous manufacturer of meat processing equipment, made a detailed introduction by videos on their vacuum stuffers, choppers, dicers, and so on. All these above provide rich materials for teaching of food technology and engineering.

'Teacher registration' facilitates teachers to manage and maintain the system. Teachers can register their accounts on the website. Having been approved by the super administrator, they can acquire the administrative authority of system through 'Teachers login' at the top right corner of the website, then carry out video editing, uploading and question-library setting.

The login teachers can upload the videos in their subjects according to the scope of the authorization. After that, they need to set questions, add options, and pick the right answers. They can also assign the number of random questions supplied to the users. For example, 20 questions have been installed for a video, and the teacher set 5 on the backstage. The system will randomly extract five of them displayed to the users.

On the page of 'Interactive teaching', the users can find quizzes of videos classified in different subjects. The system will randomly take questions from the question library. After they submit their answers, the correct options will be shown. After watching the video, users can also click 'Ready to test', at the bottom of the video-playing window, to get into the link of 'Interactive teaching'. This link is actually a small test to examine students' understanding on the video, which contains the details and key processes of certain crafts involved in the video. For example, the 'Interactive teaching' of 'Bread Slicing' video has the following test:

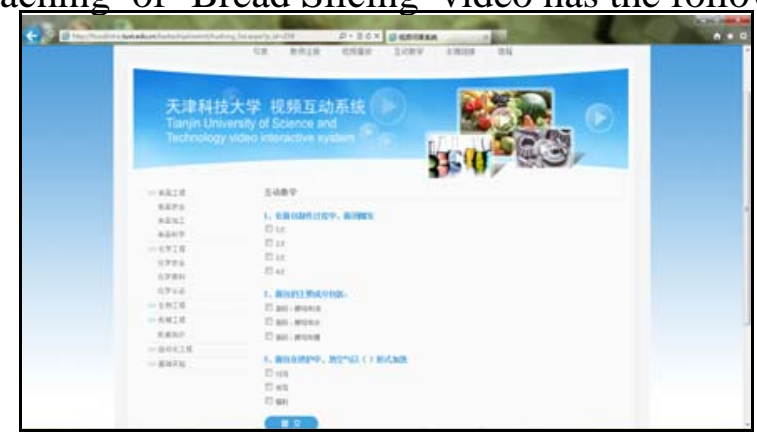

Fig.2. Test of 'Interactive teaching'

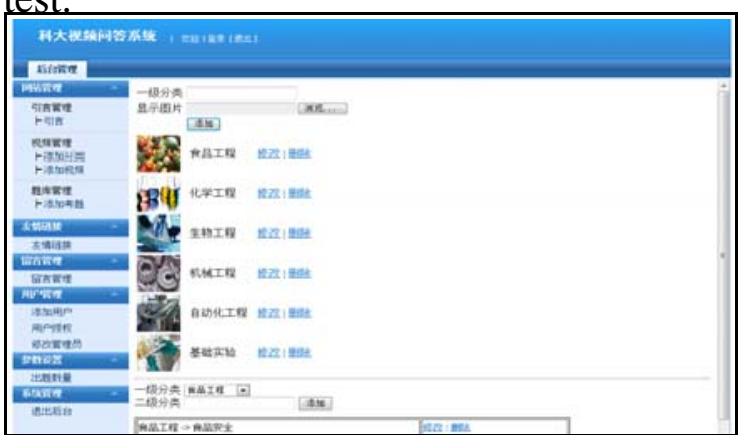

Fig.3. Super administrators’ operations

Students tick the corresponding options according to the video's content they just watched, and then click 'Submit' after finishing the test and the correct options will show up following each question. This fast-paced learning - test - grading mode enables learners to deepen their impression of knowledge in the form of entertainment and vividness. Meanwhile, teachers can stress what students need to grasp by the setting of test questions. The whole course of acquiring knowledge seems actively and lively.

On the page of 'Message board', students can communicate with teachers about any questions by a message board. And the super administrator takes charge of sorting and answering the questions. Besides, the super administrator can also set the website's fundamental parameters, manage all the videos' classification and the answers. What's more, he also approves the teachers' registration information, see Fig. 3.

\section{Technical Support}

(a)Designing environment

The operation platform supports Chinese/ English Windows XP/ Windows Vista /Windows 7 /Windows Sever 2003 /Windows Server 2008. The software development platform is VS2008 + SP1, based on MFC database. And the software development language is ASP.NET(C\#).

(b)The overall design of system

The system is a 3-tier application, that is, UI (User Interface), BLL (Business Logic Layer) and DAL (Data Access Layer).

The User Interface is the interface displayed to users when they operate the system. BLL is the operations aiming at specific matters, such as uploading, downloading, login, registration, approval, which are realized by logistical treating data. BLL is vital in the overall schema, since it connects with UI and DAL. It acts differently. For UI, it is the caller; while for DAL, it is the callee. DAL operates directly with data base, such as data appending, deleting, modifying and searching. 


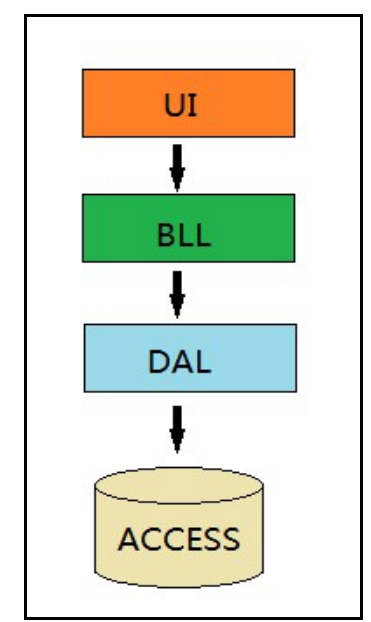

Fig.4. The schema design of the system

\section{Maintenance and Renewal}

The super administrator on backstage is responsible for the daily work of the video interactive system. The video resources and question library need to be constantly enriched and updated. Teachers are focusing persistently on the development of their disciplines, collecting related information, maintaining and updating the video interactive system.

\section{System Evaluation}

Generally speaking, we got positive feedback from students. We made a survey toward 120 students from 5 majors via emails. Sixty-two percent students supported that the video interactive system provided guidance for their automatic learning, and the data library helps to strengthen the key points in videos. Seventy-five percent students said that their queries have been answered in time. Fifty-eight percent students were satisfied with the system, and thirty percent respondents wrote that the system needed quite a few refinement.

\section{Conclusion Remarks}

The application of bilingual video resources in food education has got positive responses among students and professions. The interactive video system facilitates students' automatic e-learning, free of the constraints of time and location. Students deepen their understanding by watching well-chosen videos and answering the questions. Meanwhile, the interactive video system is a platform for communications between teachers and students. Teachers will try to perfect the system according to students' feedback, updating more videos and extending to more subjects.

\section{References}

[1] The course-based website 'Principles of Food Technology' of Tianjin University of Science and Technology http://www2.tust.edu.cn/jingpin/jp2007/spjsyl2/index.asp.

[2] The course-based website 'Introduction to food science and technology' of Tianjin University of Science and Technology http://foodintro.tust.edu.cn/.

[3] Yuanyuan JIA, Zheng ZHAO. Design and Construction of the Website of Bilingual Demonstration Course [J]. Science and Technology Innovation Herald, 200936 240-241.

[4] http://i.youku.com/u/UODUyNTA0ODA=/playlists.

[5] Zhi YU, Xuefeng BAI. Construction and application of college English teaching resources bank based on campus network [J]. Shenyang Institute of Engineering(Social Sciences), 20095 252-255.

[6] Yan LU. On the Theories and Functions of the Construction of English Video Resources[J].

Sichuan University of Science \& Engineering (Social Sciences Edition), 20111 82-84.

[7] Ying YANG, Kun QU. Investigation and Thinking of Application of Video Cases on Normal University Students' Capability Training: Take Southwest University as an example[J]. Southwest Agricultural University(Social Science Edition), 20114 172-176. 